University of Nebraska - Lincoln

DigitalCommons@University of Nebraska - Lincoln

Biological Systems Engineering: Papers and

Publications

Biological Systems Engineering

2009

\title{
Mathematical Modeling of Growth of Salmonella in Raw Ground Beef under Isothermal Conditions from 10 to $45^{\circ} \mathrm{C}$
}

Vijay K. Juneja

USDA ARS Eastern Regional Research Center

Martin Valenzuela Melendres

Research Center for Food and Development, CIAD, A. C., Hermosillo, Sonora, 83000, Mexico

Lihan Huang

USDA ARS Eastern Regional Research Center

Jeyamkondan Subbiah

University of Nebraska-Lincoln, jeyam.subbiah@unl.edu

Harshavardhan Thippareddi

University of Nebraska-Lincoln, harsha15@uga.edu

Follow this and additional works at: https://digitalcommons.unl.edu/biosysengfacpub

Part of the Biological Engineering Commons

Juneja, Vijay K.; Melendres, Martin Valenzuela; Huang, Lihan; Subbiah, Jeyamkondan; and Thippareddi, Harshavardhan, "Mathematical Modeling of Growth of Salmonella in Raw Ground Beef under Isothermal Conditions from 10 to $45^{\circ}$ C" (2009). Biological Systems Engineering: Papers and Publications. 37. https://digitalcommons.unl.edu/biosysengfacpub/37

This Article is brought to you for free and open access by the Biological Systems Engineering at DigitalCommons@University of Nebraska - Lincoln. It has been accepted for inclusion in Biological Systems Engineering: Papers and Publications by an authorized administrator of DigitalCommons@University of Nebraska Lincoln. 


\title{
Mathematical modeling of growth of Salmonella in raw ground beef under isothermal conditions from 10 to $45^{\circ} \mathrm{C}^{\mathrm{h}} \mathrm{c}$
}

\author{
Vijay K. Juneja ${ }^{\mathrm{a}, *}$, Martin Valenzuela Melendres ${ }^{\mathrm{b}}$, Lihan Huang ${ }^{\mathrm{a}}$, \\ Jeyamkondan Subbiah ${ }^{\mathrm{c}, \mathrm{d}}$, Harshavardhan Thippareddi ${ }^{\mathrm{c}}$ \\ a USDA ARS Eastern Regional Research Center, 600 East Mermaid Lane, Wyndmoor, PA 19308, USA \\ ${ }^{\mathrm{b}}$ Research Center for Food and Development, CIAD, A. C., Hermosillo, Sonora, 83000, Mexico \\ ' Department of Food Science and Technology, University of Nebraska, Lincoln, NE 68583, USA \\ d Department of Biological Systems Engineering, University of Nebraska, Lincoln, NE 68583, USA
}

\section{A R T I C L E I N F O}

\section{Article history:}

Received 9 July 2008

Received in revised form 31 December 2008

Accepted 24 January 2009

Available online $\mathrm{xxxx}$

\section{Keywords:}

Salmonella

Beef

Microbial modeling

\begin{abstract}
A B S T R A C T
The objective of this study was to develop primary and secondary models to describe the growth of Salmonella in raw ground beef. Primary and secondary models can be integrated into a dynamic model that can predict the microbial growth under varying environmental conditions. Growth data of Salmonella at nine different isothermal conditions $-10,15,20,25,28,32,35,42$, and $45^{\circ} \mathrm{C}$ were first fitted into primary models, namely the logistic, modified Gompertz, Baranyi, and Huang models. Performances of these models were evaluated by using various statistical criteria, namely mean square error (MSE), pseudo- $R^{2},-2 \log$ likelihood, Akaike's and Bayesian's information criteria. All the chosen models fitted well to the growth data of Salmonella based on these criteria. The results of statistical analysis showed that there was no significant difference in the performances of the four primary models, suggesting that the models were equally suitable for describing isothermal bacterial growth. The specific growth rates derived from each model was fitted to the Modified Ratkowsky equation, relating the specific growth rate to growth temperatures. It was also observed that the lag phase duration was an inverse function of specific growth rates. These models, if validated, can be used to construct dynamic models to predict potential Salmonella growth in raw ground beef.
\end{abstract}

Published by Elsevier B.V.

\section{Introduction}

Salmonella spp. are widely distributed in nature and are a major cause of foodborne illness in the United States. While products such as produce, fruits, and vegetables are increasingly being implicated in Salmonella foodborne illnesses, red meat and poultry remain predominant products contributing to foodborne illnesses. Improper cooking, cross contamination of cooked products and temperature abuse during processing and storage of these foods is the major cause of salmonellosis. It is known that time and temperature are two of the most important physical factors affecting the growth of bacteria in foods. Therefore, modeling the effect of temperature on the growth of Salmonella in beef products can help the industry to reliably predict and estimate potential growth of Salmonella during processing (low-temperature long-time cooking) and storage.

Traditional microbial enumeration techniques do not lend themselves for requirements of the industry to evaluate the safety of processes and

\footnotetext{
Mention of trade names or commercial products in this article is solely for the purpose of providing specific information and does not imply recommendation or endorsement by the U.S. Department of Agriculture.

* Corresponding author. Tel.: +1 215233 6500; fax: +1 2152336550.

E-mail address: vijay.juneja@ars.usda.gov (V.K. Juneja).
}

conducting microbial challenge studies are time-consuming and expensive. Mathematical microbial models are increasingly being used to evaluate the potential for growth of microorganisms in foods during processing and storage (Bovil et al., 2001). Models developed for use by the food industry and relevant regulatory agencies for risk assessment must be able to accurately estimate microbial growth under both static (isothermal) and dynamic, i.e., constantly varying conditions of temperatures. Traditionally, primary models are developed for the organism of concern under static temperature conditions. After the establishment of the primary models, the secondary models are developed to describe the growth parameters (such as rate constant) as a function of temperature over the entire range of growth temperature of the organism. After validation, the secondary models can be used to estimate and predict the growth of the organism under either isothermal or constantly varying temperature conditions, as encountered in the food industry. The empirical sigmoidal type models such as the modified Gompertz and logistic models have been used for fitting microbial growth data obtained under isothermal conditions. The biological-based Baranyi model is another model useful for modeling bacterial growth (George et al., 1996; McClure et al., 1997; Sutherland et al., 1997). Though most sigmoidal models can predict microbial growth under isothermal conditions with reasonable accuracy, they do not directly estimate the growth under dynamic (varying environmental) conditions (Huang, 2003). 
While many microbial growth models are available in the literature, the majority of them were developed using microbiological media. While these models can be used for general evaluation of potential growth of an organism of concern, their utility has been limited as they may deviate significantly in some instances of food products in the real-world food processing environment. Thus, the accuracy and reliability of the mathematical models can be improved by using the real food product.

The aim of this study was to develop primary and secondary models to describe the growth of Salmonella in beef. The performance of three commonly used primary models, namely the Baranyi, modified Gompertz, and logistic models, was directly compared. In addition, the Huang model was also assessed (Huang, 2008). The growth rate derived from each primary model was then correlated to the growth temperature to develop the secondary model using the modified Ratkowsky model. An optimal and useful dynamic model is the one that has a good fit in both primary and secondary models. Goodness-of-fit for both primary and secondary models have been used to determine the best model that can be implemented for dynamic modeling of Salmonella growth in beef.

\section{Materials and methods}

\subsection{Bacterial serotypes and preparation of test cultures}

Several Salmonella serotypes isolated from beef, chicken, turkey, food processing environment, as well as human clinical cases, were used in the study. The detailed information about the five serotypes/strains has been provided elsewhere (Juneja et al., 2007). These serotypes were stored at $-80^{\circ} \mathrm{C}$ in vials containing brain heart infusion (BHI) broth (BD Diagnostics Systems, Sparks, MD) supplemented with $15 \%$ (v/v) glycerol (Sigma Chemical Co., St. Louis, MO). To propagate the cultures, a small volume $(0.1 \mathrm{ml})$ of each culture (partially thawed at room temperature) was transferred to $10 \mathrm{ml} \mathrm{BHI}$ broth in $50 \mathrm{ml}$ tubes and incubated at $37^{\circ} \mathrm{C}$ for $24 \mathrm{~h}$ on a rotary shaker (120 rpm). A working culture for use in experiments was prepared by separately transferring $0.1 \mathrm{ml}$ of each culture to tubes of BHI broth $(10 \mathrm{ml})$ and incubating aerobically at $37^{\circ} \mathrm{C}$ for $24 \mathrm{~h}$. The resulting cultures were then maintained in BHI broth at $4{ }^{\circ} \mathrm{C}$ for 2 weeks. A new series of cultures was initiated from the frozen stock on a biweekly basis.

Fresh inocula were prepared by transferring $0.1 \mathrm{ml}$ of each of the working culture to $10 \mathrm{ml}$ of $\mathrm{BHI}$, and incubating aerobically at $37^{\circ} \mathrm{C}$ for $18 \mathrm{~h}$ to provide late stationary phase cells. The cell cultures were centrifuged (Eppendorf, model 5810R, Hamburg, Germany) washed twice with $0.1 \%$ peptone water (PW, BD Diagnostics Systems, Sparks, $\mathrm{MD}$ ), and re-suspended in PW. The final cell concentration in each culture was determined, after serial dilution with PW, by spiral plating (Model D; Spiral Biotech, Inc., Bethesda, MD), in duplicate, onto tryptic soy agar (TSA, BD Diagnostics Systems, Sparks, MD) plates and incubating at $37{ }^{\circ} \mathrm{C}$ for $48 \mathrm{~h}$. Approximately equal volumes of each culture were combined in a sterile conical tube to obtain a five-strain mixture of Salmonella cells prior to inoculation into ground beef using the procedure described below.

\subsection{Ground beef and bacterial inoculation}

Raw ground beef (95\% lean) was obtained from a local retail store. Ground beef was divided into small portions weighing $5 \mathrm{~g}$ each, placed into $30 \times 19 \mathrm{~cm}$ Stomacher 400 polyethylene bags (Spiral Biotech, Bethesda, MD) and vacuum-sealed. The divided ground beef samples were packaged into larger barrier pouches (Bell Fibre Products, Columbus, GA). Each pouch, containing 50 individually-packed 5 g ground samples, was vacuum sealed, frozen at $-36^{\circ} \mathrm{C}$, and irradiated $(25 \mathrm{kGy})$ to eliminate indigenous microflora. Random samples were tested to verify the elimination or inactivation of microflora by mixing the irradiated ground beef with PW at a 1:1 ratio. The suspension $(0.1$ and $1.0 \mathrm{ml})$ of the meat and PW slurry was directly surfaceplated onto TSA plates. The TSA plates were incubated aerobically at $37{ }^{\circ} \mathrm{C}$ for $48 \mathrm{~h}$. No colony formation indicated that microflora were eliminated in irradiated ground beef.

To inoculate ground beef, the five strains mixture of Salmonella serotypes was added $(0.1 \mathrm{ml})$ to each bag of $5 \mathrm{~g}$ thawed, irradiated ground beef. An Interscience Bag Mixer "MiniMix" (St. Nom., France) used to blend the inoculated meat for $5 \mathrm{~min}$ to ensure even distribution of the organisms in the meat sample. Bags containing irradiated meat samples mixed with $0.1 \mathrm{PW}$ were used as negative controls. The bags were compressed into a thin, uniform layer (approximately 1-2 $\mathrm{mm}$ thick) by pressing them against a flat surface, excluding most of the air, and then heat sealed using a Multivac (Model A300/16, Multivac Inc., Kansas City, MO) packaging machine, without applying a vacuum.

\subsection{Growth experiments and enumeration of bacteria}

The inoculated samples were submerged in a water bath stabilized at $10,15,20,25,28,32,35,37,42,45$ or $47^{\circ} \mathrm{C}$. At time intervals, ranging from 5 h to 7 days and dependent upon the incubation temperature, samples were periodically removed from the water bath for enumerating the bacterial counts. Samples removed from the water baths were mixed with $5 \mathrm{ml}$ of PW to obtain 1:1 slurry and then, blended in a stomacher (MiniMix Bag Mixer, Interscience, St. Nom., France) for $5 \mathrm{~min}$. The liquid portion was serially diluted and surfaceplated (in duplicate) onto TSA agar plates. The latter was incubated at $37{ }^{\circ} \mathrm{C}$ for $24 \mathrm{~h}$. An average $\mathrm{CFU} / \mathrm{g}$ of four platings of each sampling point was used to determine estimates of the growth kinetics.

Two independent experiments were performed for each temperature with duplicate samples within each replicate.

\subsection{Mathematical models}

\subsubsection{Primary models}

Modified Gompertz model (Eq. (1)) and logistic model (Eq. (2)) were used to fit the growth curves of bacteria in foods under isothermal conditions (Gibson et al., 1987).

$x(t)=x_{0}+\left(x_{\max }-x_{0}\right) \exp (-\exp (-B(t-M)))$

$x(t)=x_{0}+\frac{\left(x_{\max }-x_{o}\right)}{1+\exp (-B(t-M))}$

where: $x(t)$ is the $\log _{10}(\mathrm{CFU} / \mathrm{g})$ of cell concentration at time, $t ; x_{0}$ is the initial concentration $\left(\log _{10} \mathrm{CFU} / \mathrm{g}\right) ; B$ is maximum relative growth rate at $t=M\left(\mathrm{~h}^{-1}\right) ; M$ is time at which the absolute growth rate is maximum (h). The parameter $B$ can be used to define the specific growth rate of the isothermal growth $r_{\max }$ (McMeekin et al., 1993). Since they are sigmoidal models, both of the modified Gompertz and logistic models are more suitable to fitting growth curves complete with all three phases, including lag, exponential, and stationary phases. With incomplete growth, particularly when the stationary phase data are missing, these models may not be suitable for fitting the growth curves.

For modified Gompertz model, the specific growth rate $r_{\max }$ and lag phase duration $\lambda$ can be calculated from

$r_{\max }=\frac{x_{\max }-x_{\min }}{e} \times B, \quad \lambda=M-\frac{1}{B}$

and for the modified logistic model the specific growth rate and lag phase can be determined by

$$
r_{\max }=\frac{x_{\max }-x_{\min }}{4} \times B, \quad \lambda=M-\frac{2}{B}
$$


where $r_{\max }$ is maximum specific growth rate in terms of $\log _{10}$ cell concentration $\left(\mathrm{h}^{-1}\right)$.

The Baranyi model (Baranyi and Roberts, 1994) also was used to fit the sigmoidal bacterial growth curves at constant temperature conditions.

$y(t)=y_{0}+\mu_{\max } F(t)-\ln \left(1+\frac{e^{\mu_{\max } F(t)}-1}{e^{\left(y_{\max }-y_{0}\right)}}\right)$

where $F(t)=t+\frac{1}{v} \ln \left(e^{-v t}+e^{-h_{0}}-e^{\left(-v t-h_{0}\right)}\right) ; y(t)$ is $\ln$ CFU/g of cell concentration at time, $t ; y_{o}$ is the initial cell concentration (ln CFU/g); $y_{\max }$ is the maximum cell concentration ( $\mathrm{ln} \mathrm{CFU} / \mathrm{g}$ ); $\mu_{\max }$ is maximum specific growth rate in terms of $\ln \mathrm{CFU} / \mathrm{g}$, which is equal to $r_{\max } \ln 10$ in $\mathrm{h}^{-1} ; \nu$ is rate of increase of the limiting substrate, assumed to be equal to $\mu_{\max } ; h_{o}$ is equal to $\mu_{\max } \lambda$.

According to Baranyi and Roberts (1994), the parameter $h_{o}$ should be approximately constant in situations where the pre-inoculation history of the cells is identical. In reality, this parameter varies. Therefore, the growth data at each temperature were first fitted with the Baranyi model to obtain all four parameters $y_{o}, y_{\max }, h_{o}$, and $\mu_{\max }$. A mean value for the parameter $h_{o}$ for all growth curves was determined. After the mean $h_{o}$ values was determined, the growth data were fitted again with the Baranyi model to obtain the values of $y_{0}, y_{\max }$, and $\mu_{\max }$ for each growth curve, after fixing the value of $h_{o}$ with the mean value, $\bar{h}_{o}$.

Another recently developed growth model (Huang, 2008) was also used compared in this study. Denoting $\lambda$ as the duration of the lag phase, this model is particularly suitable for growth curves without stationary phase. The model is expressed as

$y(t)=y_{0}+\mu_{\max }\left\{t+\frac{1}{25} \ln \frac{1+\exp [-25(t-\lambda)]}{1+\exp (25 \lambda)}\right\}$.

\subsubsection{Secondary models}

After all the growth curves were fitted to the growth models, secondary models were developed. For the Baranyi and Huang models, the values of $\mu_{\max }$ were converted to $r_{\max }$ by dividing with $\ln (10)$, or 2.303. The specific growth rates $r_{\max }$ of each model were fitted to a modified Ratkowsky model (Eq. (5)) to analyze the effect of temperature on maximum growth rate $\left(r_{\max }\right.$ or $\left.\mu_{\max }\right)$ (Zwietering et al., 1991).

$r_{\max }=a\left(T-T_{\min }\right)^{2}\left\{1-\exp \left[b\left(T-T_{\max }\right)\right]\right\}$

where $T$ is the temperature $\left({ }^{\circ} \mathrm{C}\right) ; T_{\min }$ and $T_{\max }$ are the theoretical minimum and maximum temperatures $\left({ }^{\circ} \mathrm{C}\right)$ beyond which growth is not possible; $\mathrm{a}$ and $\mathrm{b}$ are regression constants.

\subsubsection{Nonlinear regression of primary models}

PROC NLIN, a nonlinear regression procedure, in the SAS package (Version 9.1, SAS Institute Inc., Cary, NC), was used to fit each individual set of growth data to the three primary models described above. The Marquardt option of the PROC NLIN procedure was used during numerical iteration to search for the parameters of each model. After nonlinear regression of the growth curves were completed, parameters for fit statistics were calculated. Assuming that the errors of the models well normally and independently distributed, the parameters for fit statistics included -2log likelihood (Eq. (6)), Akaike's information criterion (AIC, Eq. (7)), Bayesian information criterion (BIC, Eq. (8)).

-2 log likelihood:

$-2 \cdot \ln (L)=n\left[\ln \left(2 \pi \frac{S S E}{n}\right)+1\right]$

Akaike's information criterion:

$A I C=-2 \cdot \ln (L)+2 p$
Bayesian information criterion:

$B I C=-2 \ln (L)+p * \ln (n)$

where $n$ is number of observations or data points; SSE is error sum of squares in the model; and $p$ is number of parameters in the model.

Another two parameters, MSE and pseudo- $R^{2}$, were also calculated. MSE is the mean squared error of the models. In linear regression models, the quality of fit of a model can be easily expressed as the coefficient of determination, $R^{2}$. In nonlinear regression, such a measure is not readily defined. A pseudo- $R^{2}$ was to calculated to compare the quality of nonlinear models:

Pseudo-R ${ }^{2}=1-\mathrm{SS}($ Residual $) / \mathrm{SS}\left(\right.$ Total $\left._{\text {Corrected }}\right)$.

Since ln CFU/g was used in the Baranyi model and $\log _{10} \mathrm{CFU} / \mathrm{g}$ was used in both modified Gompertz and logistic models, it was not possible to compare the mean of square errors (MSE) directly from the models. The MSE values obtained form the Baranyi model will be $(\ln 10)^{2}$ times greater than those obtained from the modified Gompertz and logistics models.

\subsubsection{Secondary model}

The maximum growth rates $\left(r_{\max }\right.$ or $\left.\mu_{\max }\right)$ at different isothermal conditions estimated from each primary model were fitted to the modified Ratkowsky model (Eq. (5)). Again, the PROC NLIN procedure of SAS was used to estimate the parameters of the secondary model. MSE and pseudo- $R^{2}$ were used to evaluate and compare the performance of each secondary model, corresponding to each primary model.

\section{Results and discussion}

\subsection{Primary model fitting}

All the models were fitted to each individual growth curve satisfactorily, and shown in Fig. 1 are three examples of growth curves fitted to the modified Gompertz, logistic, Baranyi, and Huang models by nonlinear regression. The pseudo- $R^{2}$ values range from 0.948 to close to 1.00 . For the Baranyi model, the average $h_{0}$ value was 2.20 , with a standard deviation of 0.996 . This value was used to derive

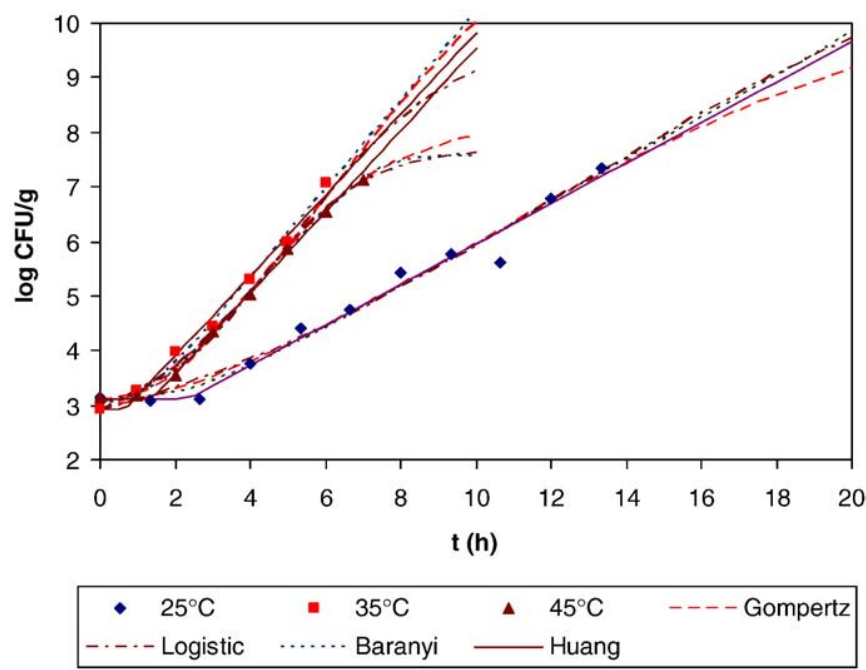

Fig. 1. Selective growth curves fitted by modified Gompertz and logistic models, Baranyi model, and Huang model. 


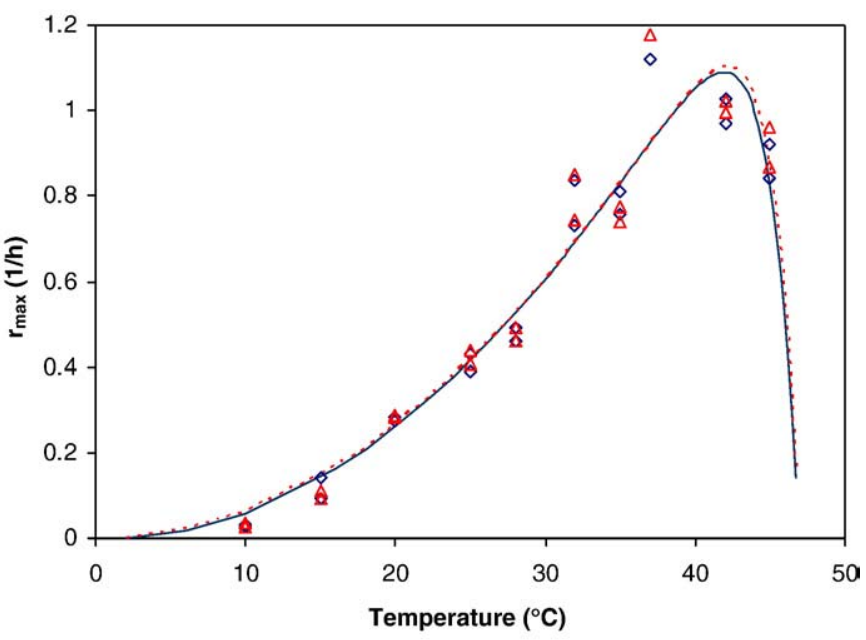

$\diamond$ Gompertz — RatG $\triangle$ Logistic $\cdots . .$. RatL

Fig. 2. Specific growth rates $\left(r_{\max }\right)$ and the Modified Ratkowsky curves derived from modified Gompertz and logistic models. (RatG - the Modified Ratkowsky curve for the Gompertz model; RatL - the Ratkowsky curve for the logistic model).

all the growth parameters for the Baranyi model. At $47{ }^{\circ} \mathrm{C}$ no growth was observed. Instead, a decline in the bacterial population was noticed. This information was particularly useful in determining the upper boundary of growth temperature for the secondary models. There were some errors in the growth curves generated at $37{ }^{\circ} \mathrm{C}$. Therefore, the growth data obtained at $37{ }^{\circ} \mathrm{C}$ were excluded in the study.

As for the fit statistics, there were no significant difference in the effect of model selection on the means of AIC, BIC, and $-2 \log$ likelihood at $\alpha=0.05$ level. Overall, the values of AIC, BIC, and $-2 \operatorname{loglikelihood~were~more~affected~by~temperature~}(p<0.001)$ than by the choice of models $(p>0.2)$. The mean AIC, BIC, and -2loglikelihood values were higher at lower temperatures (15 and $25^{\circ} \mathrm{C}$ ) and the lowest at $32{ }^{\circ} \mathrm{C}$. There were not significant difference in the means of AIC, BIC, and -2loglikelihood values at other temperature conditions. The mean MSE values were higher at 15 and $25{ }^{\circ} \mathrm{C}$, while there were no significant differences in the means of MSE at other temperature conditions. Contrary to MSE, the pseudo$R^{2}$ was the lowest at 15 and $25{ }^{\circ} \mathrm{C}$, but basically the same at other temperature conditions. The statistically analysis of AIC, BIC, $-2 \log$ likelihood, MSE, and pseudo- $R^{2}$ values suggest that all of the growth models were equally suitable for fitting the growth curves and with similar accuracy. The results of statistical analysis by ANOVA also indicated that the experimental conditions at 15 and $25{ }^{\circ} \mathrm{C}$ might have affected the growth data generation at these temperatures, which led to relatively higher systematic errors, which might explain the relative higher AIC, BIC, -2 loglikelihood, and MSE values, and relatively lower pseudo- $R^{2}$ at these two temperature conditions.

\subsection{Growth parameters and secondary model}

\subsubsection{Specific growth rate}

The specific growth rates $\left(r_{\max }\right)$ derived from each primary model were fitted to the Modified Ratkowsky equation (Eq. (5)). Since a decline, at a rate of $0.37 \log \mathrm{CFU} / \mathrm{g} / \mathrm{h}$, in the population of Salmonella was observed at $47{ }^{\circ} \mathrm{C}$, this information was proven critical in determining the maximum growth temperature for the secondary models. Fig. 2 shows the Modified Ratkowsky curves derived from modified Gompertz and logistic models. For the modified Gompertz model, the Modified Ratkowsky equation takes the form of

$r_{\max }=7.18 \times 10^{-4}(T-0.93)^{2}\{1-\exp [0.464(T-46.96)]\}, \quad R^{2}=0.990$.

For the modified logistic model, the Modified Ratkowsky equation can be expressed as

$r_{\max }=6.98 \times 10^{-4}(T-0.55)^{2}\{1-\exp [0.506(T-46.96)]\}, \quad R^{2}=0.990$.

It is evident, from both Fig. 2 and Eqs. (10) and (11), that there was no substantial difference in the specific growth rate derived from the modified Gompertz and logistic models. The lowest growth temperatures determined from the Gompertz and logistic models were 0.93 and $0.55^{\circ} \mathrm{C}$, respectively. The highest growth temperature determined from both models was $46.96{ }^{\circ} \mathrm{C}$, which was in consistence with the experimental observation. The maximum and minimum temperatures derived from the modified Ratkowsky models were "theoretical maximum and minimum temperatures", and may not present the true maximum and minimum temperatures of the bacteria. Nevertheless, the theoretical maximum and minimum temperatures are necessary for the modified Ratkowsky models.

Fig. 3 shows that the data of specific growth rates obtained from the Baranyi and Huang models, and the Modified Ratkowsky curves derived from the two models. For the Baranyi model, the Modified Ratkowsky equation takes the form of

$r_{\max }=6.44 \times 10^{-4}(T-0.38)^{2}\{1-\exp [0.3837(T-46.97)]\}, \quad R^{2}=0.986$.

For the Huang model, the Modified Ratkowsky equation becomes

$r_{\max }=5.75 \times 10^{-4}(T+0.09)^{2}\{1-\exp [0.4329(T-46.96)]\} \quad R^{2}=0.988$.

The lowest growth temperature determined from the Baranyi and Huang models were very close to 0 , and the highest temperature was slightly below $47^{\circ} \mathrm{C}$.

Statistically, there was no significant difference among the means of the raw data of specific growth rates derived from the primary models used in this study. All Modified Ratkowsky equations for specific growth rates suggest that the minimum growth temperature for Salmonella in beef was between $0-1{ }^{\circ} \mathrm{C}$, and the maximum growth temperature was around $47{ }^{\circ} \mathrm{C}$. Therefore, the Modified Ratkowsky equation for $r_{\max }$ may be used to estimate specific growth rates of

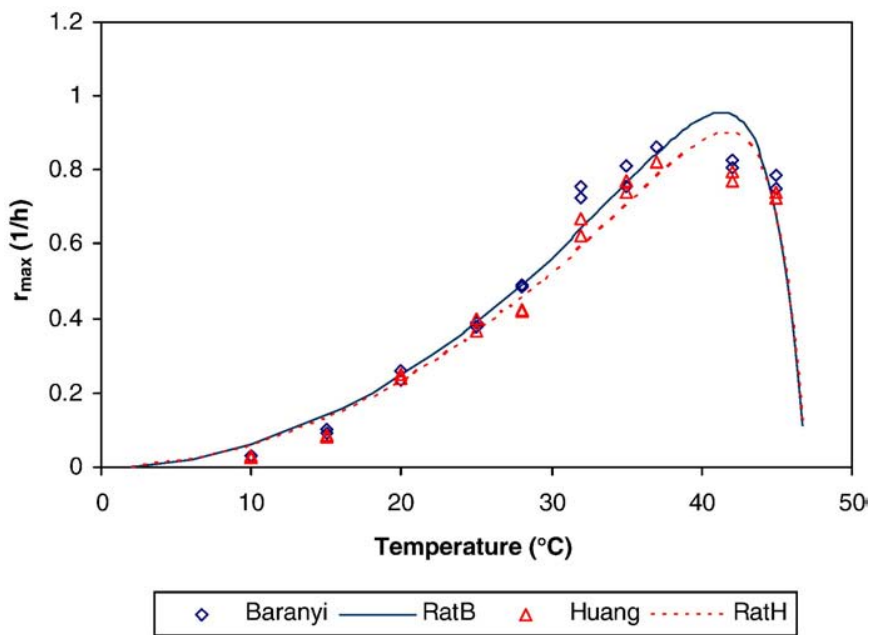

Fig. 3. Specific growth rates $\left(r_{\max }\right)$ and the Ratkowsky curves derived from Baranyi and Huang models (RatB - the Modified Ratkowsky curve for the Baranyi model; RatH the Modified Ratkowsky curve for the Huang model). 
Salmonella in beef between $0-47^{\circ} \mathrm{C}$. Since the data were collected at temperatures between $10-47^{\circ} \mathrm{C}$, cautions should be exercised when applying the model at temperatures below $10^{\circ} \mathrm{C}$. The rate calculated from the Modified Ratkowsky models was slightly higher than the experimental observation, making the model more conservative when they are used in risk assessment.

However, there were significant differences among the means of specific growth rates $\left(r_{\max }\right)$ calculated from different Modified Ratkowsky equations. At temperatures between 2 to $46.75{ }^{\circ} \mathrm{C}$, there was no significant difference between the means of the calculated $r_{\text {max }}$ derived from the modified Gompertz and logistic models at $\alpha=0.05$ level. Nor was there a difference between the means of the calculated $r_{\max }$ derived from the Baranyi and Huang models. No difference was detected between the calculated $r_{\max }$ derived from the modified Gompertz and Baranyi models. However, the mean of the calculated $r_{\max }$ derived from the modified logistic model was significantly higher than that from the Huang model.

\subsubsection{Lag phase duration}

For Baranyi model, the estimation of lag phase duration $(\lambda)$ was directly tied to $h_{0}$ and $\mu_{\max }$, and can be directly calculated from the Modified Ratkowsky equation (Eq. (12)) using the following relationship

$\lambda=\frac{h_{0}}{2.303 r_{\max }}, \quad$ or, $\quad \lambda=\frac{0.955}{r_{\max }}$.

The modified Gompertz and logistic models were merely curvefitting equations that could describe the general trend of the bacterial growth. These two models would perform well if the growth curves were well defined and complete with all the phases. Since all the growth curves obtained from this study did not contain the stationary phases, the estimation of the lag phase duration for some of the growth curves were apparently not accurate. The calculated lag phase duration for three of the growth curves at lower temperatures was negative, and apparently not accurate. Therefore, these three data points were excluded for further analysis.

For the rest of the growth curves with positive lag phase duration, the inverse relationship between $\lambda$ and $r_{\max }$ was also observed for the lag phase derived from the modified Gompertz and logistic model

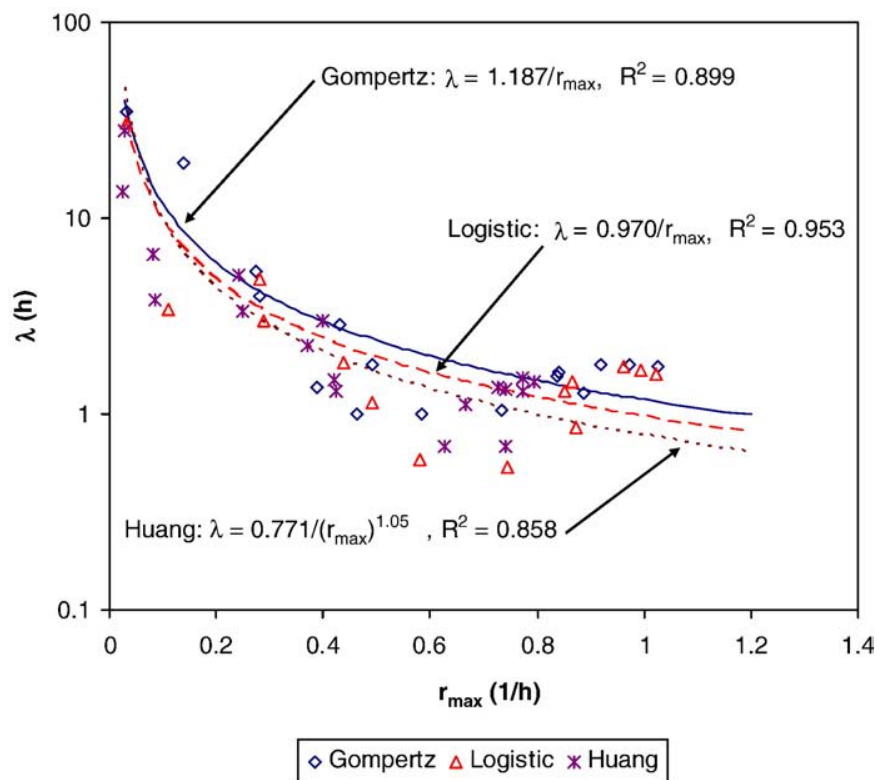

Fig. 4. Relationship between the lag phase duration $(\lambda)$ and specific growth rate derived from Gompertz, logistic, and Huang models.
(Fig. 4). For modified Gompertz model, the lag phase duration cane calculated from

$\lambda=\frac{1.187}{r_{\max }}, \quad R^{2}=0899$.

For modified logistic model, $\lambda$ can be calculated from

$\lambda=\frac{0.970}{r_{\max }}, \quad R^{2}=0.953$

For the Huang model, the lag phase duration was explicit in the model and was directly calculated for each growth curve. However, the lag phase duration was also related to specific growth rates (Fig. 4), and the relationship between $\lambda$ and $r_{\max }$ derived from the Huang model can be expressed as

$\lambda=\frac{0.771}{r_{\max }^{1.05}}, \quad R^{2}=0.858$

A recurring debate relates to which of the growth models were better for describing the bacterial growth. It has been argued that the empirical models such as modified Gompertz and logistic models were not as preferred as the more mechanistic models such as the Baranyi model. This study directly compared the two empirical models with more mechanistic Baranyi and Huang models. The results revealed that there were no significant difference among the four models, which suggests that any of them can be used to describe the bacterial growth under isothermal conditions. For the empirical models, it would be more desirable to obtain complete, i.e., from the lag to stationary phases, growth curves prior to curve fitting to improve the accuracy of the derived secondary growth parameters $\left(r_{\max }\right.$ and $\left.\lambda\right)$. The mechanistic models such as Baranyi and Huang models are more versatile, capable of fitting curves without stationary phases. The Baranyi model, however, involves a two-step process. In the first step, the $h_{0}$ of each individual curves is determined. After each individual curve was analyzed, an average $\left(\bar{h}_{o}\right)$ of all $h_{0}$ values is taken. It is necessary to analyze each individual curve again with $h_{0}$ fixed to the average $\bar{h}_{0}$. As a result, the analysis with the Baranyi model would more time-consuming than that of the other models. For the Huang model, the specific growth rate $\left(r_{\max }\right)$ and the duration of the lag phase $(\lambda)$ of each growth curve can be obtained in one-step.

The ultimate purpose of developing primary and secondary models is to estimate and predict bacterial growth under dynamic conditions. For implementation of dynamic models, the primary and secondary models have to be integrated and solved numerically. There can be several other factors that may affect the performance of a dynamic model. For instance, Huang (2003) reported that the starting values for some parameters and step size can affect the performance of the dynamic model. Thus, it is important to validate the dynamic models with new growth data of Salmonella collected in beef under dynamically changing temperatures.

These dynamic models can be used to predict potential Salmonella growth in beef and beef products. These models can be used to develop safe holding temperatures for beef and to set critical limits in HACCP plans for beef to prevent or reduce potential growth of Salmonella. Further, the utility of these models include use in cases of deviations from the critical limits within HACCP plans, and to evaluate potential risk of Salmonella growth in beef.

\section{References}

Baranyi, J., Roberts, T.A., 1994. A dynamic approach to predicting bacterial growth in food. Int. J. Food Microbiol. 23, 277-294.

Bovil, R.A., Bew, J., Baranyi, J., 2001. Measurements and predictions of growth for Listeria monocytogenes and Salmonella during fluctuating temperature. II. Rapidly changing temperatures. Int. J. Food Microbiol. 67, 131-137. 
George, S., Richardson, L.C.C., Peck, M.W., 1996. Predictive models of the effect of temperature, $\mathrm{pH}$ and acetic and lactic acids on the growth of Listeria monocytogenes. Int. J. Food Microbiol. 32, 73-90.

Gibson, A.M., Bratchell, N., Roberts, T.A., 1987. The effect of sodium chloride and temperature on the rate and extent of growth of Clostridium botulinum type $\mathrm{A}$ in pasteurized pork slurry. J. Appl. Bacteriol. 62, 479-490.

Huang, L., 2003. Dynamic simulation of Clostridium perfringens growth in cooked ground beef. Int. J. Food Microbiol. 87, 217-227.

Huang, L., 2008. Growth Kinetics of Listeria monocytogenes in Broth and Beef Frankfurters-Determination of Lag Phase Duration and Exponential Growth Rate under Isothermal Conditions. J. Food Sci. 73, E235-E242.

Juneja, V.K., Melendres, M.V., Huang, L., Gumudavelli, V., Subbia, J., Thippareddi, H., 2007. Modeling the effect of temperature on growth of Salmonella in chicken. Food Microbiol. $24,328-335$
McClure, P.J., Beaumont, A.L., Sutherland, J.P., Roberts, T.A., 1997. Predictive modeling of growth of Listeria monocytogenes. The effects on growth of $\mathrm{NaCl}, \mathrm{pH}$, storage temperature and $\mathrm{NaNO}_{2}$. Int. J. Food Microbiol. 34, 221-232.

McMeekin, T.A., Olley, J.N., Ross, T., Ratkowsky, D.A., 1993. Predictive Microbiology: Theory and Application. John Wiley \& Sons, Inc., New York, NY. 340 pp.

Sutherland, J.P., Bayliss, A.J., Braxton, D.S., Beaumount, A.L., 1997. Predictive modeling of Escherichia coli 0157:H7: inclusion of carbon dioxide as a fourth factor in a pre-existing model. Int. J. Food Microbiol. 37, 113-120.

Zwietering, M.H., De Koos, J.T., Hasenack, B.E., De Wit, J.C., van't Riet, K., 1991. Modeling of the bacterial growth as a function of temperature. Appl. Environ. Microbiol. 57, 1094-1101. 